University of Nebraska - Lincoln

DigitalCommons@University of Nebraska - Lincoln

Faculty Publications in Computer \& Electronics Electrical \& Computer Engineering, Department Engineering (to 2015)

2008

\title{
Performance Analysis of Multi-hop IEEE 802.11 DCF Backhaul Networks
}

\author{
Puttipong Mahasukhon \\ Hamid Sharif \\ University of Nebraska-Lincoln, hsharif@unl.edu \\ Michael Hempel \\ University of Nebraska-Lincoln, mhempel2@unl.edu
}

Ting Zhou

Wei Wang

See next page for additional authors

Follow this and additional works at: https://digitalcommons.unl.edu/computerelectronicfacpub

Part of the Computer Engineering Commons

\begin{abstract}
Mahasukhon, Puttipong; Sharif, Hamid; Hempel, Michael; Zhou, Ting; Wang, Wei; and Wysocki, Tadeusz, "Performance Analysis of Multi-hop IEEE 802.11 DCF Backhaul Networks" (2008). Faculty Publications in Computer \& Electronics Engineering (to 2015). 12.

https://digitalcommons.unl.edu/computerelectronicfacpub/12
\end{abstract}

This Article is brought to you for free and open access by the Electrical \& Computer Engineering, Department of at DigitalCommons@University of Nebraska - Lincoln. It has been accepted for inclusion in Faculty Publications in Computer \& Electronics Engineering (to 2015) by an authorized administrator of DigitalCommons@University of Nebraska - Lincoln. 


\section{Authors}

Puttipong Mahasukhon, Hamid Sharif, Michael Hempel, Ting Zhou, Wei Wang, and Tadeusz Wysocki 
IEEE International Conference on Wireless \& Mobile Computing, Networking \& Communication Published in Networking and Communications, 2008. WIMOB '08. IEEE International Conference on Wireless and Mobile Computing, 12-14 Oct. 2008 Page(s):69 - 74

Digital Object Identifier 10.1109/WiMob.2008.123

\title{
Performance Analysis of Multi-hop IEEE 802.11 DCF Backhaul Networks ${ }^{\dagger}$
}

\author{
Puttipong Mahasukhon, Hamid Sharif, Michael Hempel, \\ Ting Zhou, Wei Wang, and Tadeusz Wysocki \\ Computer and Electronics Engineering Department, University of Nebraska-Lincoln \\ \{pmahasukhon, hsharif, mhempel,tzhou,wwang,twysocki\}@unlnotes.unl.edu
}

\begin{abstract}
Because of the wide deployment of 802.11 equipments in the past decade, current applications are not limited anymore to only single Access Points (AP) deployments for indoor usage, but have been extended to multi-hop networks to fulfill the need of high speed connectivity in mobile environments, where the analysis of multi-hop networks is extremely complicated. The behavior of an AP is dependent not only on its neighbors' behavior, but also on the behavior of other hidden nodes. In this paper, we provide an accurate and verified multi-hop wireless backhaul analysis for performance of IEEE 802.11 $D C F$ in terms of the channel throughput using a static channel error rate. The model is based on analysis of a single hop communications for evaluating the multihop wireless backhaul networks. We utilized our existing 3.5-mile Federal Railroad Administration (FRA) test bed on the BNSF railroad track in Nebraska to validate the model. Our field measurements and simulation results show that our proposed model is accurate.
\end{abstract}

\section{Introduction}

In recent years, IEEE 802.11 WLANs [1] have become the dominant technology for wireless networking. As the demand for broadband mobile data services increases, larger coverage areas increasingly become indispensable. Due to the limitation of the protocol, single-hop transmission range is capable of at most a couple of miles and is not sufficient for mobile subscribers. The cost for last mile technologies, like IEEE 802.16e [2], is still relatively expensive because of the equipment itself and associated fees for

\footnotetext{
$\dagger$ This work was supported by a grant from the Federal Railroad Administration (FRA) under Grant 25-1105. The support provided to us by BNSF and Union Pacific Railway.
}

operating in licensed frequency bands. Thus, multi-hop wireless networks become a potential solution for mobile users.

The performance analysis of IEEE 802.11 Distribution Coordination Function (DCF) has been covered in several research efforts through simulation, experiment, or modeling. There is considerable interest in modeling the performance of saturated single-hop networks. Most prominently, the work from [3] has gained high acceptance due to first introducing a Markov chain model for analyzing the binary exponential backoff mechanism of 802.11 DCF. However, the model was designed to characterize the backoff procedure under an ideal case, using unlimited number of retries in an error-free channel. Based on this work, many enhancements have been done focusing on different aspects. The enhancement models under saturation load have been proposed in [4].

However, multi-hop backhaul networks require the capability of analyzing the performance under finite load conditions. However, only a few research activities have focused on modeling under finite load. Although their models are also based on Markov chain, all of them modeled it in different ways. In [5], [6], and [7], after Distributed InterFrame Space (DIFS), if a node has a packet to send, they suddenly go to slot 0 in the first backoff state, whereas the standard states that the backoff procedure will be invoked when the medium is busy or a transmission fails. In [8], they forgot the fact that the probability of having a packet to transmit in an idle channel needs to be considered for the DIFS period. Besides, all of previous works have been verified only in simulation.

The paper is organized as follows. The analytical model is described in Section 2, including throughput analyses of a single-hop network. Multi-hop wireless backhaul in the test bed is presented in Section 3. Finally, ns-2 simulation results and test bed results validating the model are presented in Section 4. Concluding remarks are given in Section 5. 


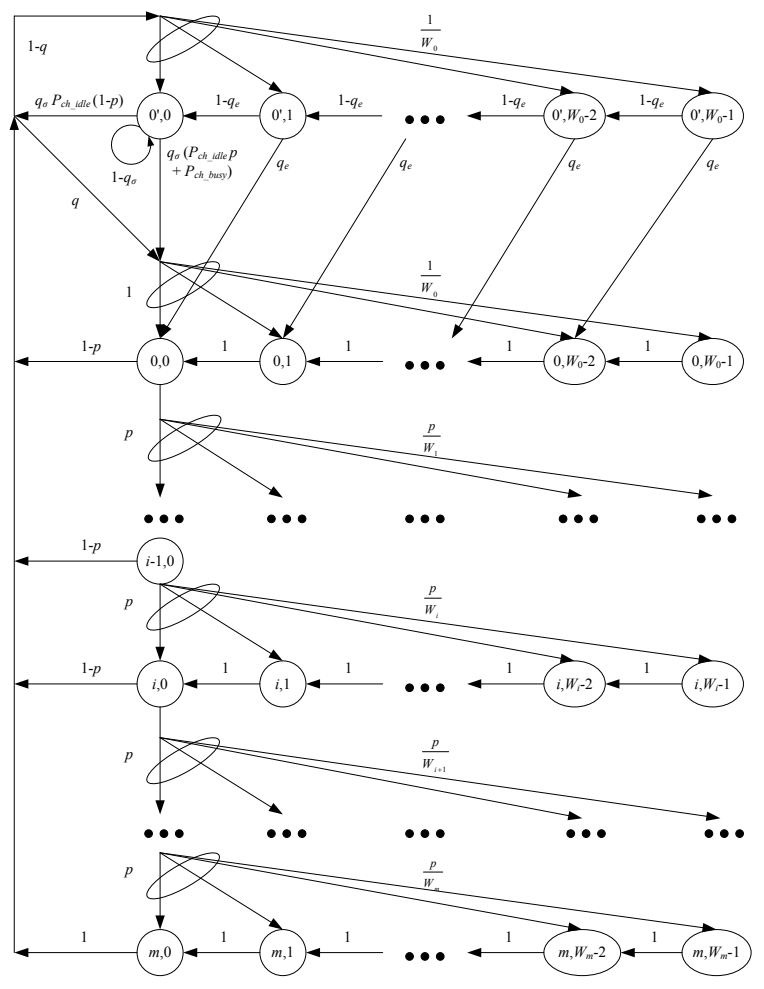

Figure 1. Markov chain model

\section{Markov chain model}

This section provides a detailed MAC throughput analysis based on a Markov chain model [3]. Let $b(t)$ be the stochastic process representing the backoff window size for a given station at slot time $t$. The states $s(t)$ of a transmit node are modeled by a pair of states $(i, k)$ as shown in Fig. 1. The backoff state, $i$, starts at 0 and is increased by 1 for every time a transmission fails up to a maximum value $m$ '. We define the contention window size $W$ at the backoff stage $i$ for convenience.

$$
\begin{array}{cc}
W_{i}=2^{i} W_{0} & i \leq m^{\prime} \\
W_{i}=2^{m^{\prime}} W_{0} & i>m^{\prime}
\end{array}
$$

$W_{0}$ is the initial contention window size $\left(C W_{\min }+1\right)$. After each unsuccessful transmission, $W_{i}$ is doubled up to $2^{m} W_{0}=C W_{\max }+1$. However, it is possible that $i$ could be larger than $m$ ' if the maximum retransmission count $m$ is greater than $m^{\prime}$, while the $C W$ will be held after that.

Let $P_{t r\{n\}}$ be the probability that there is at least one transmission in the considered slot time. Since a fixed number of $n$ stations contend on the channel and node $x$ transmits with probability $\tau_{x}$, we obtain (2). Let $P_{t r\{n-1\}}$ be the probability that a busy channel slot occurs, given that the node in question is idle.

$$
\begin{aligned}
& P_{t r\{n\}}=1-\prod_{x=1}^{n}\left(1-\tau_{x}\right) \\
& P_{t r\{n-1\}}=1-\prod_{y=1, y \neq x}^{n}\left(1-\tau_{y}\right)
\end{aligned}
$$

Therefore, $1-P_{t r\{n\}}$ is the probability that there is no transmission in the considered slot time. Also, 1-P $P_{t r\{n-1\}}$ is the probability that an idle channel slot occurs while the node in question is idle. Let $P_{c}$ be the probability that a transmitted packet collides.

$$
P_{c}=1-\prod_{y=1, y \neq x}^{n}\left(1-\tau_{y}\right)
$$

Let $P_{s\{n\}}$ be the probability that a transmission is successful. From the same manner, $P_{s\{n-1\}}$ is the probability that a successful transmission in a channel slot occurs while the node in question is idle.

$$
\begin{aligned}
P_{s\{n\}}= & \frac{\sum_{x=1}^{n}\left(\tau_{i} \prod_{y=1, y \neq x}^{n}\left(1-\tau_{y}\right)\right)}{P_{t r\{n\}}} \\
P_{s\{n-1\}}= & \frac{\sum_{y=1, y \neq x}^{n}\left(\tau_{y} \prod_{z=1, z \neq y}^{n}\left(1-\tau_{z}\right)\right)}{P_{t r\{n-1\}}}
\end{aligned}
$$

Let $P_{e}$ be the probability that a transmission is successful. The expressions of probability of any frame error can be written as a function of bit error rate (BER) in (5) and the probabilities of specific type of frame error can be obtained in (6)

$$
P_{e}=1-(1-B E R)^{\text {sizeRTS+sizeCTS+sizeDATA+sizeACK }}
$$

$$
\begin{aligned}
& P_{e}^{r t s}=1-(1-B E R)^{\text {sizerTS }} \\
& P_{e}^{c t s}=\left((1-B E R)^{\text {sizeRTS }}\right) \cdot\left(1-(1-B E R)^{\text {sizeCTS }}\right) \\
& P_{e}^{\text {data }}=\left((1-B E R)^{\text {sizeRTS sizecTS }}\right) \cdot\left(1-(1-B E R)^{\text {sizeDATA }}\right) \\
& P_{e}^{\text {ack }}=\left((1-B E R)^{\text {sizeRTS+sizeCTS+sizeDATA }}\right) \cdot\left(1-(1-B E R)^{\text {sizeACK }}\right)
\end{aligned}
$$

Let $p$ be the unsuccessful transmission probability, through packet collision or the presence of a bit error.

$$
p=P_{c}+\left(1-P_{c}\right) P_{e}
$$


Furthermore, the following equations of the time overhead are a result of frame errors.

$$
\begin{aligned}
& T_{e}^{r t s}=T_{r t s}+T_{c t s}^{\text {timeout }}+\delta+D I F S \\
& T_{e}^{c t s}=T_{r t s}+T_{c t s}+2 \delta+S I F S+D I F S \\
& T_{e}^{\text {data }}=T_{r t s}+T_{c t s}+H+E[P]+T_{a c k}^{\text {timeout }}+3 \delta+2 S I F S+D I F S \\
& T_{e}^{\text {ack }}=T_{r t s}+T_{c t s}+H+E[P]+A C K+4 \delta+3 S I F S+D I F S
\end{aligned}
$$

Let $H=P H Y_{h d r}+M A C_{h d r}$ be the packet header and $\delta$ be the propagation delay. $T_{s}$ is the average time the channel is sensed busy because of a successful transmission, and $T_{c}$ is the average time the channel is sensed busy by the stations during a collision. The equations for $T_{s}$ and $T_{c}$ can be expressed below. In the basic access case we obtain

$$
\begin{aligned}
& T_{s}^{b a s}=H+E[P]+A C K+2 \delta+S I F S+D I F S \\
& T_{c}^{b a s}=H+E\left[P^{*}\right]+\delta+D I F S
\end{aligned}
$$

where $E\left[P^{*}\right]$ is the average length of the longest packet payload involved in a collision. In the case all packets have the same fixed size, $E\left[P^{*}\right]=E[P]=P$. For the RTS/CTS access method, collisions can occur only on RTS frames, we obtain

$$
\begin{aligned}
& T_{s}^{r s s}=R T S+C T S+H+E[P]+A C K+4 \delta+3 S I F S+D I F S \\
& T_{c}^{r s}=R T S+\delta+D I F S
\end{aligned}
$$

Each time slot, $\sigma$, has a probability of $1-P_{t r\{n-1\}}$ being idle, $1-P_{t r\{n-1\}} P_{s\{n-1\}}\left(1-P_{e}\right)$ of having a successful transmission without any packet error, $P_{t r\{n-1\}} P_{s\{n-1\}} P_{e}^{x x x}$ of having a packet $x x x$ error, and $P_{t r\{n-1\}}\left(1-P_{s\{n-1\}}\right)$ of having a collision. Therefore, the average slot time can be calculated as

$$
\begin{aligned}
\bar{\sigma}= & \left(1-P_{t r\{n-1\}}\right) \sigma+P_{t r\{n-1\}} P_{s\{\{-1\}}\left(1-P_{e}\right) T_{s} \\
& +P_{t r\{n-1\}} P_{s\{n-1\}}\left(P_{e}^{r t s} T_{e}^{r t s}+P_{e}^{c t s} T_{e}^{c t s}+P_{e}^{d a t a} T_{e}^{d a t a}+P_{e}^{a c k} T_{e}^{a c k}\right) \\
& +P_{t r\{n-1\}}\left(1-P_{s\{n-1\}}\right) T_{c}
\end{aligned}
$$

Let $q_{\sigma}$ be the packet arrival probability in a slot time with mean $\lambda$ and let $q$ be the probability of having at least one packet to send. Equation (12) shows the probability of a node remaining idle and the transition probability of entering the backoff procedure. The backoff procedure will be invoked for a station to transmit a frame when the medium is busy, a transmission fails, or there are still some packets left in the queue after a transmission ends.

$$
\begin{aligned}
& P\left\{0^{\prime}, 0 \mid 0^{\prime}, 0\right\}=1-q_{\sigma} \\
& P\left\{0, k \mid 0^{\prime}, 0\right\}=\frac{q_{\sigma}}{W_{0}}\left(P_{c h \_b u s y}+P_{\text {ch_idle }} p+P_{\text {ch_idle }}(1-p) q\right)
\end{aligned}
$$

The packet arrival probability in a slot time, the probability of channel idle for a DIFS period, and the probability of channel busy somewhere within a DIFS can be written as

$$
\begin{aligned}
& q_{\sigma}=1-e^{-\lambda \sigma} \\
& P_{c h \_ \text {idle }}=\left(1-P_{t r\{n-1\}}\right)^{D I F S / \sigma} \\
& P_{c h \_b u s y}=1-P_{c h \_i d l e}
\end{aligned}
$$

The transition probabilities inside the backoff stage are given in (14). At the beginning of each slot time, the backoff time decrements when the station senses that the channel is idle. A new packet following a successful transmission starts with a backoff stage 0 , and the backoff interval is initially uniformly chosen. At the maximum backoff stage, no matter if the transmission succeeds or fails, the node will discard that packet and if there are some packets left in the queue, it will re-enter the backoff stage for a new packet. If an unsuccessful transmission occurs at the backoff stage, the backoff stage increases, and the new backoff value is uniformly chosen.

$$
\begin{array}{ll}
P\{i, k \mid i, k+1\}=1 & i \in(0, m) ; k \in\left(0, W_{i}-2\right) \\
P\{0, k \mid i, 0\}=(1-p) q / W_{0} & i \in(0, m-1) ; k \in\left(0, W_{0}-1\right) \\
P\{0, k \mid m, 0\}=q / W_{0} & k \in\left(0, W_{0}-1\right) \\
P\{i, k \mid i-1,0\}=p / W_{i} & i \in(1, m) ; k \in\left(0, W_{i}-1\right)
\end{array}
$$

A backoff interval is selected from the range $\left[0, W_{0^{-}}\right.$ 1] immediately after the end of every transmission even if no additional transmission is currently queued to avoid channel capture. The state $S_{0^{\prime}, k}$ is the special backoff state. The transition probabilities of entering the special backoff stage are given in (15).

$$
\begin{aligned}
& P\left\{0^{\prime}, k \mid i, 0\right\}=(1-p)(1-q) / W_{0} \\
& P\left\{0^{\prime}, k \mid m, 0\right\}=(1-q) / W_{0} \\
& P\left\{0^{\prime}, k \mid 0^{\prime}, 0\right\}=q_{\sigma} P_{\text {ch_idle }}(1-p)(1-q) / W_{0}
\end{aligned}
$$

In the special backoff state, the backoff time also decrements when the station senses that the channel is idle. If there are some packets arriving during this time, it will switch first to the regular backoff state. The 
transition probabilities from inside the special backoff stage are given in (16).

$$
\begin{array}{ll}
P\left\{0^{\prime}, k \mid 0^{\prime}, k+1\right\}=1-q_{e}=e^{-\lambda \bar{\sigma}} & k \in\left(0, W_{0}-2\right) \\
P\left\{0, k \mid 0^{\prime}, k+1\right\}=q_{e}=1-e^{-\lambda \bar{\sigma}} & k \in\left(0, W_{0}-2\right)
\end{array}
$$

$$
\text { Let } b_{i, k}=\lim _{t \rightarrow \infty} P\{s(t)=i, b(t)=k\} \text { when } i \in\left(0^{\prime}, m\right)
$$
and $k \in\left(0, W_{i}-1\right)$ be the stationary distribution of the chain. We now show how to obtain a closed-form solution for this Markov chain. First, note that

$$
b_{i-1,0} \cdot p=b_{i, 0} \rightarrow b_{i, 0}=p^{i} \cdot b_{0,0} \quad i \in(1, m)
$$

By means of these relations from the chain regularities, the backoff state $S_{i, k}$ of each $i \in(1, m)$ can be rewritten as

$$
b_{i, k}=\frac{W_{i}-k}{W_{i}} \cdot b_{i, 0} \quad i \in(1, m) \quad k \in\left(1, W_{i}-1\right)
$$

Then, trying to express all the values $b_{0, k}, b_{i, k}$, and $b_{0, k}$ in (19) below as functions of the value $b_{0,0}$.

$$
1=\sum_{i=0}^{m} \sum_{k=0}^{W_{i}-1} b_{i, k}+\sum_{k=0}^{W_{0}-1} b_{0^{\prime}, k}
$$

By making use of $(1-p) \sum_{i=0}^{m-1} b_{i, 0}+b_{m, 0}=b_{0,0}$, the probability $\tau$ that a station transmits in a randomly chosen slot time can be expressed as

$$
\tau=\sum_{i=0}^{m} b_{i, 0}+q_{\sigma} b_{0^{\prime}, 0}=\frac{1-p^{m+1}}{1-p} \cdot b_{0,0}+q_{\sigma} b_{0^{\prime}, 0}
$$

Also from (3) and (7), the probability of unsuccessful transmission can be rewritten as

$$
p=1-\prod_{y=1, y \neq x}^{n}\left(1-\tau_{y}\right)\left(1-P_{e}\right)
$$

Therefore, (20) and (21) represent a nonlinear system with the two unknown variables $\tau$ and $p$, which can be solved using numerical results. After we obtain the value of $\tau$, we know the value of $P_{t r}, P_{s}$, and $P_{c}$. Then, we are able to express the normalized throughput, $S$, as the fraction of channel time being used for the actual data transmission.

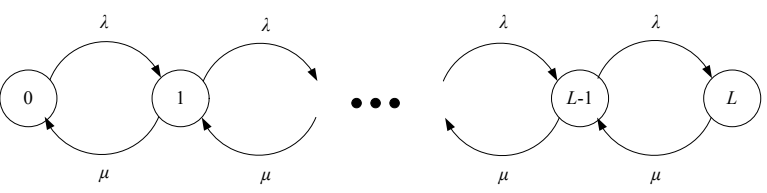

Figure 2. $M / G / 1 / L$ state transition diagram (Single node case)

$$
S=\frac{E[\text { Pay load transmitted in a slot time }]}{E[\text { Length of a slot time }]}
$$

Now we can express normalized throughput $S$ as follows

$$
S=\frac{P_{t r} P_{s}\left(1-P_{e}\right) E[P]}{\left[\begin{array}{l}
\left(1-P_{t r}\right) \sigma+P_{t r} P_{s}\left(1-P_{e}\right) T_{s} \\
+P_{t r} P_{s}\left(P_{e}^{r t s} T_{e}^{r t s}+P_{e}^{c t s} T_{e}^{c t s}+P_{e}^{d a t a} T_{e}^{d a t a}+P_{e}^{a c k} T_{e}^{a c k}\right) \\
+P_{t r}\left(1-P_{s}\right) T_{c}
\end{array}\right]}
$$

Note that (23) is applicable for both basic and RTS/CTS access methods, since when we use it to calculate the throughput performance for the basic access method we can simply take out all parameters related to RTS/CTS.

The state transition diagram of the queue model is depicted in Fig. 2. Each state represents the number of packets stored in the queue. Let $\lambda$ be the packet arrival rate, $\mu$ be the packet processing rate and $L$ be the queue length. Using the $M / G / 1 / L$ queuing model, the probability $1-q$ of having no packet for transmission, which is the steady state distribution of $b_{0}$, is equal to

$$
1-q=b_{0}=\frac{1-\lambda / \mu}{1-(\lambda / \mu)^{L+1}}
$$

Because of multi-hop backhaul nature, each hop throughput relies on its previous hop throughput. Therefore, the previous hop throughput is considered as the packet arrival rate for the next hop and so on until the last hop throughput analysis is achieved.

\section{Multi-hop backhaul in our FRA test bed}

In order to validate the Markov chain model, we tested the throughput per hop in our FRA test bed on BNSF railroad track in Nebraska. This segment features several turns, but also straight segments of track. Some parts are surrounded by dense foliage, while others are open. Our FRA test bed is about 3.5 miles between Crete, NE, and Berks, NE, and includes 8 outdoor APs. The longest interval between adjacent 
TABLE 1. IEEE 802.11a System Parameters Used in Test Bed, Analysis, and Simulations

\begin{tabular}{lll}
\hline \hline \multicolumn{1}{c}{ Parameters } & \multicolumn{1}{c}{ Test Bed } & \multicolumn{1}{c}{$\begin{array}{c}\text { Analysis \& } \\
\text { Simulation }\end{array}$} \\
\hline Antenna Type & Directional & Directional \\
Antenna Height & 10 meters & $\begin{array}{l}10 \text { meters } \\
9 \mathrm{dBi}\end{array}$ \\
Antenna Gain & $9 \mathrm{dBi}$ & $5.8 \mathrm{GHz}$ Band \\
Frequency & $5.8 \mathrm{GHz}$ Band & $25 \mathrm{dBm}$ \\
Transmit Power & $25 \mathrm{dBm}$ & Free Space Model \\
Propagation Model & - & $-91 \mathrm{dBm}$ \\
Noise Floor & $-91 \mathrm{dBm}$ & $1400 \mathrm{Bytes}$ \\
Packet Payload & 1400 Bytes & 28 Bytes \\
MAC Header & 28 Bytes & 38 Bytes \\
ACK Packet & 38 Bytes & 24 Bytes \\
PHY Preamble & 24 Bytes & $54 \mathrm{Mbps}$ \\
Data Rate & Rate Adaptation & $9 \mu \mathrm{s}$ \\
Slot Time & $9 \mu \mathrm{s}$ & $16 \mu \mathrm{s}$ \\
SIFS & $16 \mu \mathrm{s}$ & $34 \mu \mathrm{s}$ \\
DIFS & $34 \mu \mathrm{s}$ & 15 \\
$C W_{\text {min }}$ & 15 & 1023 \\
$C W_{\text {max }}$ & 1023 & 7 \\
Short Retry Limit & 7 & 4 \\
Long Retry Limit & 4 & 884.9 meters \\
AP8 - AP7 & 884.9 meters & 494.9 meters \\
AP7 - AP6 & 494.9 meters & 734.3 meters \\
AP6 - AP5 & 734.3 meters & 198.7 meters \\
AP5 - AP4 & 198.7 meters & \\
\hline \hline
\end{tabular}

APs is 1915 meters, while the shortest one is 198 meters. Each AP deployed in our test bed is Strix Access/One OWS with two $802.11 \mathrm{a}$ radios, two $802.11 \mathrm{~b} / \mathrm{g}$ radios, and one Ethernet port. IEEE $802.11 \mathrm{a}$ [9] is chosen for establishing the wireless backhaul network for interconnecting each AP, while $802.11 \mathrm{~b}$ is used for client access. To obtain further distances between APs, directional patch antennas facing towards adjacent APs in both forward and backward links have been employed. The channel assignment has been designed to use a sequence of non-overlapping channels to avoid co-channel interference between adjacent links.

In the test bed, we utilized a data generator connected to AP8 via a 100Mbps Ethernet connection to transmit packets to a destination station that was also connected to another AP via a $100 \mathrm{Mbps}$ Ethernet connection, hence utilizing only the wireless backhaul links. The default data frame size is 1400 bytes and the experiments use 802.11 unicast data frames. After the traffic of $25 \mathrm{Mbps}$ was generated from AP8, we captured all packets to analyze UDP throughput measurements for each hop individually. We started capturing packets at AP7 first and then we moved the destination station to AP6, AP5, and AP4, to achieve a total of 4 hops in our tests, measuring not only UDP throughput, but also TCP, FTP, and HTTP throughput. The results are reported in [11].

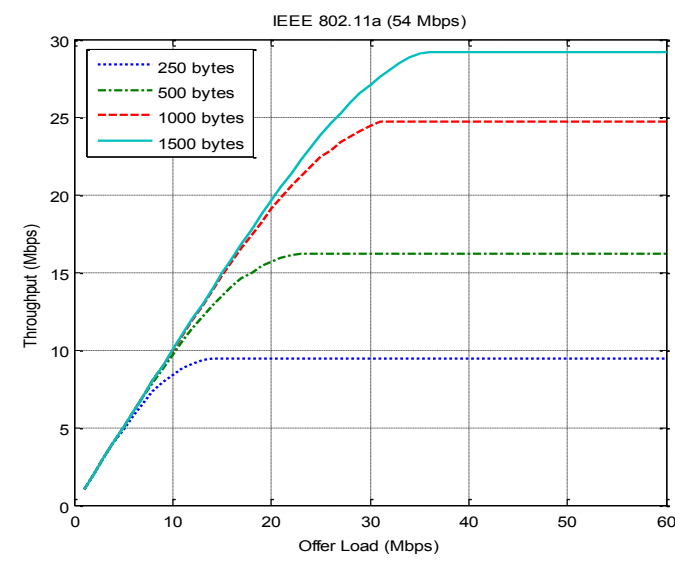

Figure 3. Throughput vs Offer load in IEEE 802.11 a system.
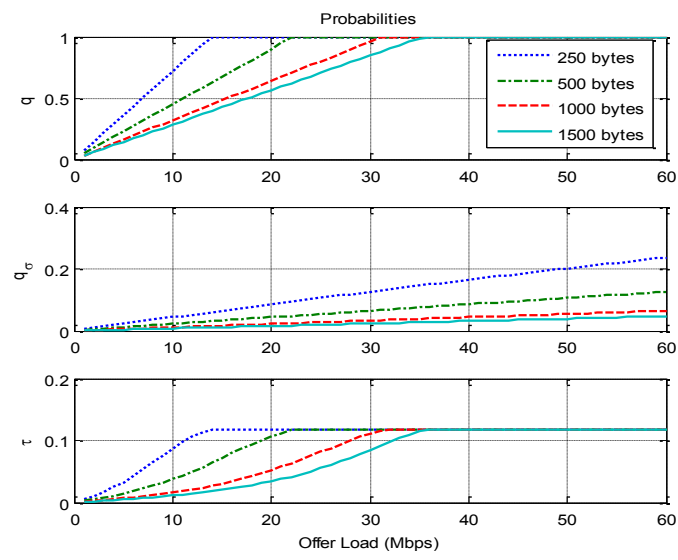

Figure 4. Probability of some packets in the queue, probability of arrival packet in a slot time, and probability of transmission related to the results in Figure 3.

\section{Results comparison}

We have developed an ns-2 model [12] that incorporates the capability to simulate multi-interface AP nodes similar to the APs we have in the actual test bed. Some radios are used for backhaul interconnection, whereas others are used for client access. Consequently, we can simulate a scenario close to our test bed in ns-2 by utilizing the protocol and channel specific parameters that are presented in Table 1.

The single-hop throughput, with different packet sizes, as a function of offer load is shown in Fig. 3, whereas the corresponding probability of packets present in the queue, probability of packets arriving during a slot time, and the probability of transmission are shown in Fig 4. 
In Fig. 5, the multi-hop throughput is processed by the proposed model and compared to the results from both ns- 2 simulation and test bed measurements. The results from the test bed is lowest due to the fact that, as earlier mentioned, some parts of our test bed are surrounded by dense foliage, and rate adaptation was applied to optimize the overall performance. Therefore, some of the packets were not transmitted at the highest data rate in order to avoid packet error. According to our records, 35 percents of all total packets were transmitted at a data rate of $54 \mathrm{Mbps}$, whereas 50 percents were sent at $48 \mathrm{Mbps}$. The current analytical model does not account for rate adaptation, and instead suffers a throughput reduction due to the higher packet loss rate. Over multiple hops the number of lost packets accumulates, resulting in a reduction of end-to-end throughput with increasing number of hops. In contrast, the ns-2 simulation, which currently does not model obstructions from foliage, outperforms others, including the test bed.

\section{Conclusion}

In summary, we have proposed an alternative approach to modeling IEEE 802.11 DCF under finite load in order to study the performance in multi-hop wireless backhaul networks. It is an accurate model and accounts for realistic network operation, such as nonsaturated networks, a finite number of retransmission attempts, and channel error. The model verification is carried out by comparing the theoretical results to ideal ns-2 simulation results and real equipment performance measurements.

The current analytical results are for multi-hop backhaul applications and in the future, this work will be extended for mesh networks.

\section{References}

[1] IEEE Standard 802.11, "Part 11: Wireless LAN medium access control (MAC) and physical layer (PHY) specifications," 1999.

[2] IEEE Standard 802.16e, "Part 16: Air Interface for Fixed and Mobile Broadband Wireless Access Systems," 2005.

[3] G. Bianchi, "Performance analysis of the IEEE 802.11 distributed coordination function," IEEE JSAC, vol. 18, no. 3, pp. 535-547, March 2000.

[4] S. Ci, H. Sharif, and P. Mahasukhon, "Evaluating saturation throughput performance of the IEEE 802.11 MAC under fading channels," in Proc. IEEE Broadnet'05, Boston, vol. 1, pp. 726-731, October 2005.

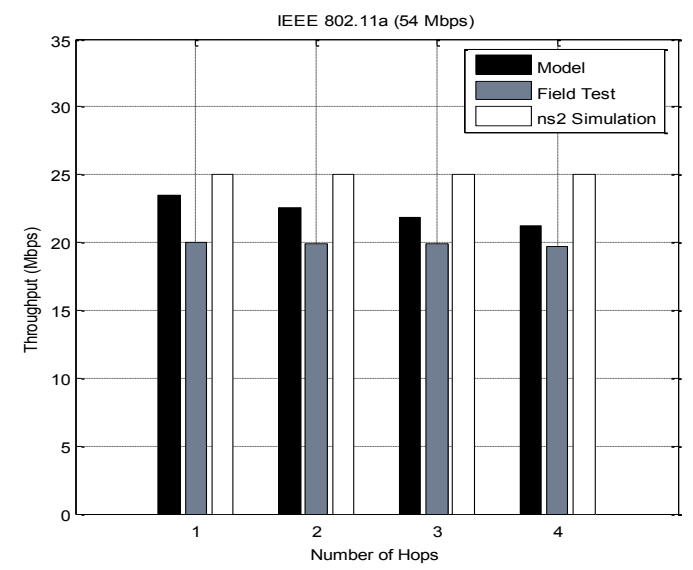

Figure 5. Results comparison: analytical model vs test bed results and ns-2 simulation.

[5] J. V. Sudarev, L. B. White, and S. Perreau, "Performance analysis of $802.11 \mathrm{CSMA} / \mathrm{CA}$ for infrastructure networks under finite load condition." in LANMAN'05 workshop, Greece, September 2005.

[6] P. P. Pham, S. Perreau, and A. Jayasuriya, "New crosslayer design approach to ad hoc networks under Rayleigh fading," IEEE JSAC, vol. 23, no. 1, pp. 28-39, January 2005.

[7] K. Duffy, D. Malone, and D. J. Leith, "Modeling the 802.11 distributed coordination function in non-saturated conditions," IEEE Communications Letters, vol. 9, no. 8, pp. 715-717, August 2005.

[8] F. A.-Shabdiz and S. Subramaniam, "Analytical models for single-hop and multi-hop ad hoc networks," in Proc. IEEE Broadnet'04, 2004.

[9] IEEE Standard 802.11a, "Part 11: Wireless LAN medium access control (MAC) and physical layer (PHY) specifications: high speed physical layer in the $5 \mathrm{GHz}$ band," 1999.

[10] M. Hempel, H. Sharif, T. Zhou, and P. Mahasukhon, “A wireless test bed for mobile 802.11 and beyond," in Proc. of IWCMC'06, Vancouver, Canada, pp. 1003-1008, July 2006.

[11] T. Zhou, H. Sharif, M. Hempel, P. Mahasukhon, W. Wang, and S. Ci, "Performance Study of a Mobile Multi-hop 802.11 Railway Network Using Passive Measurement," submitted for publication.

[12] Ns-2 (Network simulator 2) [Online]. Available: http://www.isi.edu/nsnam/ns/. 